\title{
VARIANCE UPPER BOUNDS AND A PROBABILITY INEQUALITY FOR DISCRETE $\alpha$-UNIMODALITY
}

Abstract. Variance upper bounds for discrete $\alpha$-unimodal distributions defined on a finite support are established. These bounds depend on the support and the unimodality index $\alpha$. They increase as the unimodality index $\alpha$ increases. More information about the underlying distributions yields tighter upper bounds for the variance. A parameter-free Bernstein-type upper bound is derived for the probability that the sum $S$ of $n$ independent and identically distributed discrete $\alpha$-unimodal random variables exceeds its mean $E(S)$ by a positive value $n t$. The bound for $P\{S-n \mu \geq n t\}$ depends on the range of the summands, the sample size $n$, the unimodality index $\alpha$ and the positive number $t$.

1. Introduction. Unimodality concepts for distributions are well known in the continuous case. Olshen and Savage (1970) generalized this concept to $\alpha$-unimodality. They defined a continuous random variable (r.v.) $X$ to be $\alpha$-unimodal (about the origin), $\alpha \geq 0$, if and only if there exists some r.v. $Y$ such that $X={ }^{d} U^{1 / \alpha} Y$, where $U$ is a uniform r.v. on $(0,1)$ independent of $Y$. The $\alpha$-unimodal distributions have been further studied by many authors (see Abouammoh and Mashhour (1983), Alamatsaz (1985), Dharmadhikari and Jogdeo (1986) and reference therein).

Upper bounds on variance represent an important target, since they have applications in many areas of statistics such as variance estimation and stochastic process; see Dharamaddhikari and Joag-Dev (1989) and references therein. They proved that if $X$ is a continuous r.v. having $\alpha$-unimodal distribution about $M, 0 \leq X \leq 1$ and $\mu=E(X)$, then

$$
(\alpha+2) \operatorname{Var}(X) \leq \mu(\alpha+1+2 M)-(\alpha+2) \mu^{2}-M .
$$

2000 Mathematics Subject Classification: Primary 62E10.

Key words and phrases: discrete unimodality; variance; upper and lower bounds; probability inequality. 
Among other results they show that the upper bound for the variance of an $\alpha$-unimodal distribution on $[0,1]$ is $(\alpha+1)^{2} /\left(4(\alpha+2)^{2}\right)$, which yields Jacobson's bound of $\frac{1}{9}$ when $\alpha=1$ (see Jacobson (1969)).

For continuous independent and identically distributed (i.i.d) unimodal r.v.'s $X_{1}, \ldots, X_{n}$ with bounded support, Young et al. (1988) derived a parameter-free Berstein upper bound for $P\{S-n \mu \geq n t\}$, where $S=$ $\sum_{i=1}^{n} X_{i}$.

Abouammoh et al. (1994) defined the discrete $\alpha$-unimodality concept for $\alpha \geq 0$ as follows:

Definition 1.1. A discrete r.v. $X$ is called $\alpha$-unimodal about $a, a \in I$, $I$ is the set of integers, if its probability mass function (p.m.f.) $\left(p_{n}\right)_{n=-\infty}^{\infty}$ satisfies

$$
\begin{array}{ll}
(\alpha-n+a) p_{n} \geq(1-n+a) p_{n-1}, & n \leq a, \\
(\alpha+n-a) p_{n} \geq(1+n-a) p_{n+1}, & n \geq a .
\end{array}
$$

Note that if $\left(p_{n}\right)_{n=-\infty}^{\infty}$ is $\alpha$-unimodal about $a$ and $\beta>\alpha$ then $\left(p_{n}\right)_{n=-\infty}^{\infty}$ is $\beta$-unimodal about $a$. Consequently, all $\alpha$-unimodal distributions with $\alpha<1$ are unimodal distributions. For $\alpha \geq 1$, Abouammoh et al. (1994) give the following characterization.

TheOREM 1.1. The p.m.f. $\left(p_{n}\right)_{n=-\infty}^{\infty}$ with characteristic function (ch.f.) $p(t)$ is $\alpha$-unimodal about $n=a$ iff

$$
q(t)=\frac{\left\{\alpha+a\left(1-e^{i t}\right)\right\} p(t)+i\left(1-e^{i t}\right) p^{\prime}(t)}{\alpha}
$$

and

$$
r(t)=\frac{\left\{\alpha+a\left(e^{-i t}-1\right)\right\} p(t)+i\left(e^{-i t}-1\right) p^{\prime}(t)}{\alpha}
$$

are ch.f.'s.

Also Abouammoh and Mashhour (1994) proved that if the r.v. $X$ has discrete $\alpha$-unimodal distribution about $a$, defined on the support $\{0,1, \ldots, N\}$, with mean $\mu$, then

$$
\begin{aligned}
(\alpha+2) \operatorname{Var}(X) \leq & -(\alpha+2) \mu^{2}+[(\alpha+1) N+2 a] \mu \\
& -N a+\alpha[\min \{\mu, N-\mu\}] .
\end{aligned}
$$

This is a discrete version of (1.1).

For any discrete r.v. defined on the support $\{0,1, \ldots, N\}$ with mean $\mu$, Muilwijk (1966) showed that $\operatorname{Var}(X) \leq(N-\mu) \mu$. The right side becomes maximum when $\mu=N / 2$. Hence, if the end points of the support of any discrete r.v. are known, an upper bound for its variance may be found as

$$
\operatorname{Var}(X) \leq N^{2} / 4 \text {. }
$$


Note that equality holds when $X$ assumes the values 0 or $N$ each with probability $1 / 2$. Thus, the value $N^{2} / 4$ represents the maximum upper bound for the variance of any discrete r.v. on the support $\{0,1, \ldots, N\}$.

For a discussion of the practical relevance of variance bounds see Navard et al. (1993) and the literature mentioned there. See also Rayner (1975) and Seaman et al. (1985).

The purpose of the present article is twofold. First, in Section 2, we extend the result (1.4) due to Abouammoh and Mashhour (1994), to establish upper bounds for the variance of discrete $\alpha$-unimodal r.v.'s, sharper than that given by (1.5). These upper bounds are discrete versions for their continuous counterpart due to Dharmadhikari and Joag-Dev (1989). The new results, with $\alpha=1$, yield some interesting upper bounds for the variance of discrete unimodal r.v.'s on finite supports. The latter case, with $\alpha=1$, corresponds to results due to Young et al. (1988) in the continuous case. Next, in Section 3, our results of Section 2 are applied to get an upper bound for $P\{S-n \mu \geq n t\}$ when the $X_{i}$ 's are discrete i.i.d. $\alpha$-unimodal r.v.'s.

2. Variance upper bounds. Let $X$ be a discrete r.v. on the support $\{0,1, \ldots, N\}$. If $N=1$, then $X$ is a strongly unimodal r.v. Furthermore, it assumes only the values 0 or 1 with probabilities $q$ and $p$ respectively, $p+q=1$. One may easily deduce that $\operatorname{Var}(X)=p q \leq 1 / 4$, where equality holds when $p=q=1 / 2$. Therefore, our results henceforth will mainly concern $N \geq 2$. Assume that $X$ has an $\alpha$-unimodal, $\alpha \geq 1$, distribution about the modal value $a$. By Theorem 1.1, let $X_{1}$ and $X_{2}$ be the discrete r.v.'s whose ch.f.'s are $q(t)$ and $r(t)$ respectively. One can easily show that $X_{1}$ and $X_{2}$ are defined on the supports $S_{1}=\{0,1, \ldots, N+1\}$ and $S_{2}=$ $\{-1,0, \ldots, N\}$ respectively. Put $\mu_{1}=E\left(X_{1}\right)$ and $\mu_{2}=E\left(X_{2}\right)$. Then the ch.f.'s $q(t)$ and $r(t)$ given by (1.2) and (1.3) yield

$$
\mu_{1}=\mu_{2}=\frac{(\alpha+1) \mu-a}{\alpha}
$$

(see Abouammoh and Mashhour (1994)). In view of the fact that $S_{1}$ is non-negative and the support $\{-N-1,-N,-N+1, \ldots, 0\}$ of $X_{2}-N$ is non-positive, one may deduce that $\mu_{1}>0$ and $\mu_{2}<N$.

Hence the expectation $\mu=E(X)$ must satisfy

$$
\frac{a}{\alpha+1}<\mu<\frac{a+N \alpha}{\alpha+1} .
$$

On the other hand (1.4) implies

$$
(\alpha+2) \operatorname{Var}(X) \leq-(\alpha+2) \mu^{2}+[(\alpha+1) N+2 a] \mu-N a+\frac{\alpha N}{2},
$$

since $\min \{\mu, N-\mu\} \leq N / 2$. 
The right side of (2.3) attains its maximum when

$$
\mu=\frac{(\alpha+1) N+2 a}{2(\alpha+2)} .
$$

This value of $\mu$ satisfies the restriction (2.2) iff $\alpha^{2}+2 \alpha>|2 a /(N-1)|$. Thus, obviously, the latter condition is satisfied for all $\alpha \geq 1$.

Based on the above discussion, one may substitute $\mu$ from (2.4) into (2.3) to get

THEOREM 2.1. If $X$ is a discrete $\alpha$-unimodal r.v. about a on the support $\{0,1, \ldots, N\}$, then

$$
\operatorname{Var}(X) \leq \frac{N^{2}(\alpha+1)^{2}-4 a(N-a)}{4(\alpha+2)^{2}}+\frac{\alpha N}{2(\alpha+2)} .
$$

Theorem 2.2. Let $X$ be a discrete $\alpha$-unimodal r.v. about a on the support $\{0,1, \ldots, N\}$.

(a) If $a=0$ or $a=N$, then

$$
\operatorname{Var}(X) \leq \frac{N^{2}(\alpha+1)^{2}}{4(\alpha+2)^{2}}+\frac{\alpha N}{2(\alpha+2)} .
$$

(b) If $N$ is even and $a=N / 2$, then

$$
\operatorname{Var}(X) \leq \frac{\alpha N(N+2)}{4(\alpha+2)} .
$$

(c) If $N$ is odd and $a=(N \pm 1) / 2$, then

$$
\operatorname{Var}(X) \leq \frac{\alpha N(N+2)}{4(\alpha+2)}+\frac{1}{4(\alpha+2)^{2}} .
$$

Pr o of. Part (a) is immediate from Theorem 2.1; recall that the bound (2.5) becomes maximum when $a=0$ or $N$. Parts (b) and (c) follow by setting $a=N / 2$ and $a=(N \pm 1) / 2$ respectively in (2.5).

Also setting $\mu=N / 2$ in (2.3) yields the same upper bound (2.7), for any modal value $a$.

TheOREM 2.3. Let $X$ be a discrete $\alpha$-unimodal r.v. on the support $\{0,1$, $\ldots, N\}$. If $\mu=E(X)=N / 2$, then

$$
\operatorname{Var}(X) \leq \frac{\alpha N(N+2)}{4(\alpha+2)} .
$$

For $\alpha=1$, the p.m.f. $\left(p_{n}\right)$ is unimodal on the support $\{0,1, \ldots, N\}$, and one gets 
Corollary 2.4. Let the p.m.f. $\left(p_{n}\right)$ have mode a and variance $\sigma^{2}$.

(a) If $a=0$ or $a=N$, then

$$
\sigma^{2} \leq \frac{N^{2}}{9}+\frac{N}{6}
$$

(b) If $1 \leq a \leq N-1$, then

$$
\sigma^{2} \leq \frac{1}{9}\left[N^{2}-a(N-a)\right]+\frac{N}{6} .
$$

(c) If $N$ is even and $a=N / 2$, then

$$
\sigma^{2} \leq \frac{N^{2}}{12}+\frac{N}{6}=\operatorname{Var}(U)
$$

where $U$ is the discrete r.v. uniformly distributed on the same support.

(d) If $N$ is odd and $a=(N+1) / 2$, then

$$
\sigma^{2} \leq \frac{(N+1)^{2}}{12} .
$$

Corollary 2.5. If the p.m.f. $\left(p_{n}\right)$ is unimodal with mean $\mu=N / 2$, then

$$
\sigma^{2} \leq \frac{N^{2}}{12}+\frac{N}{6}
$$

Note that (2.10) represents the discrete version of Jacobson's theorem (1969). Moreover (2.12) is the discrete version of a result due to Seaman et al. (1987). Abouammoh and Mashhour (1994) introduced some different restrictions on the mean and mode which guarantee the upper bounds (2.12) and (2.14).

REMARK 1. For $\alpha=1$, the upper bounds (2.10)-(2.13) are at least as sharp as the bound $N^{2} / 4$ given by (1.5). Thus, the unimodality property of $X$ yields lower upper bounds for its variance. As $\alpha$ gets larger than one, the situation is quite different. Regarding that (2.6) is increasing in $\alpha$, it is not expected that the bound (2.6) will be less than $N^{2} / 4$ for all values of $\alpha$. Investigation of the bounds (2.6) and (2.7) shows that both are less than $N^{2} / 4$ when $\alpha<N$. Consequently, as $N$ gets larger our results assign to more $\alpha$-unimodal r.v.'s on the support $\{0,1, \ldots, N\}$ sharp upper bounds for their variances. Practically, free-parameter upper bounds are established based on the known information about $X$. For instance, if the only known information about $X$ is that it is $\alpha$-unimodal about a specified mode and $\alpha<N$, our results yield sharper upper bounds than $N^{2} / 4$, as given by (2.6)-(2.8). Otherwise, when $\alpha \geq N$, the only available upper bound is $N^{2} / 4$ which describes the case of any discrete r.v. on the same support.

3. Probability inequality. Let $X_{1}, \ldots, X_{n}$ be i.i.d. discrete $\alpha$-unimodal r.v.'s where $X_{i}$ has the support $\{0,1, \ldots, N\}$ with $E\left(X_{i}\right)=\mu$ and $\operatorname{Var}\left(X_{i}\right)$ 
$=\sigma^{2}$. Let $S=X_{1}+\ldots+X_{n}$ and $\bar{X}=S / n$. We derive a parameter-free upper bound for the probability

$$
P\{\bar{X}-\mu \geq t\}=P\{S-E(S) \geq n t\},
$$

where $t>0$. One method is attributed to S. N. Bernstein (see Young et al. (1989) and references therein). According to that method

$$
P\{S-n \mu \geq n t\} \leq E[\exp \{c(S-n \mu-n t)\}],
$$

for any positive constant $c$. Since the $X_{i}$ 's are independent,

$$
E[\exp \{c(S-n \mu-n t)\}]=\exp (-c n t) \prod_{i=1}^{n} E\left[\exp \left\{c\left(X_{i}-\mu\right)\right\}\right],
$$

where $c>0$. The new upper bound is derived by bounding

$$
E\left[\exp \left\{c\left(X_{i}-\mu\right)\right\}\right]
$$

from above and then minimizing the resulting bound with respect to $c$. The following lemma uses the results of Theorems 2.1 and 2.2 to establish an upper bound on (3.2).

Lemma 3.1. Let $X$ be a discrete $\alpha$-unimodal r.v. about a, with mean $\mu$ and variance $\sigma^{2}$, on the support $\{0,1, \ldots, N\}$. Let $Z=X-\mu$. Then

$$
E[\exp \{c Z\}] \leq \exp [\theta\{\exp (c N)-c N-1\}],
$$

where $c$ is an arbitrary positive constant and $\theta=\theta(\alpha, a, N)$ represents an upper bound for $(\sigma / N)^{2}$.

Proof. We have

$$
\begin{aligned}
E[\exp (c Z)] & =1+\frac{\sum_{r=2}^{\infty} c^{r} E\left(Z^{r}\right)}{r !} \leq 1+\frac{\sum_{r=2}^{\infty} c^{r} \sigma^{2} N^{r-2}}{r !} \\
& =1+\frac{\sigma^{2}}{N^{2}} \frac{\sum_{r=2}^{\infty}(c N)^{r}}{r !}=1+\frac{\sigma^{2}}{N^{2}}[\exp (c N)-c N-1] \\
& \leq 1+\theta[\exp (c N)-c N-1] \leq \exp [\theta\{\exp (c N)-c N-1\}] .
\end{aligned}
$$

Now, we establish the main results for the case of i.i.d. discrete $\alpha$ unimodal r.v.'s with finite support.

Theorem 3.2. Let $X_{1}, \ldots, X_{n}$ be i.i.d. discrete $\alpha$-unimodal r.v.'s on the support $\{0,1, \ldots, N\}$. Let $E\left(X_{i}\right)=\mu$ and $S=X_{1}+\ldots+X_{n}$. Then

$$
P\{S-n \mu \geq n t\} \leq \exp \left\{\frac{n t}{N}-\left(\frac{n t}{N}+n \theta\right) \ln \left(1+\frac{t}{N \theta}\right)\right\},
$$

where

$$
\theta=\theta(\alpha, a, N)=\frac{(\alpha+1)^{2}-4 a(N-a)}{4(\alpha+2)^{2}}+\frac{\alpha}{2(\alpha+2) N}
$$


when $X_{i}$ is $\alpha$-unimodal about the specified mode $a$, and

$$
\theta=\theta(\alpha, N)=\frac{(\alpha+2)^{2}}{4(\alpha+2)^{2}}+\frac{\alpha}{2(\alpha+2) N}
$$

when $X_{i}$ is $\alpha$-unimodal about some mode such that $\mu=N / 2$.

Proof. Let $Z_{i}=X_{i}-\mu$. Then for any positive constant $c$,

$$
\begin{aligned}
P\{S-n \mu \geq n t\} & \leq E[\exp \{c(S-n \mu-n t)\}] \\
& \leq \exp (-n c t) \prod_{i=1}^{n} E\left[\exp \left(c Z_{i}\right)\right] \\
& \leq \exp [(-n c t)+n \theta\{\exp (c N)-c N-1\}],
\end{aligned}
$$

from Lemma 3.1.

Let $g(c)=n[-c t+\theta\{\exp (c N)-c N-1\}]$. Then $g(c)$ is minimum at $c=(1 / N) \ln (1+t /(N \theta))$. Hence the result.

Corollary 3.3. Let $X$ be a discrete $\alpha$-unimodal r.v. on the support $\{0,1, \ldots, N\}$. Then for any $t>0$,

$$
P\{X-\mu \geq t\} \leq \exp \left\{\frac{t}{N}-\left(\frac{t}{N}+\theta\right) \ln \left(1+\frac{t}{N \theta}\right)\right\},
$$

where $\theta$ is given by (3.4) or (3.5) of Theorem 3.2.

Note that the upper bound in (3.6) does not depend on any parameter of $X$ other than the range $N$, the unimodality index $\alpha$ and the mode value $a$. This feature makes (3.6) very applicable in real-data situation.

REMARK 2. If $X$ is a discrete r.v. on the support $\{0,1, \ldots, N\}$, then (3.3) holds with $n=1$ and $\theta=1 / 4$. That is,

$$
P\{X-\mu \geq t\} \leq \exp \left\{\frac{t}{N}-\left(\frac{t}{N}+\frac{1}{4}\right) \ln \left(1+\frac{4 t}{N}\right)\right\} .
$$

Finally, inequality (2.2) seems to be interesting in the following sense:

(i) It provides lower and upper bounds for the mean of any discrete r.v. $\alpha$-unimodal, $\alpha \geq 1$, about a modal value $a$ on the support $\{0,1, \ldots, N\}$. Also, it represents a discrete version for its most recent continuous counterpart due to Dharmadhikari and Joag-Dev (1989).

(ii) As a consequence of (2.2),

$$
X-\frac{a+N \alpha}{\alpha+1} \geq t \quad \text { implies } \quad X-\mu \geq t .
$$

Hence an upper bound for $P\{X-a \geq t+\alpha(N-a) /(\alpha+1)\}$ may be obtained by (3.6). 
Acknowledgements. The author is greatly indebted to the referee, whose comments and suggestions have greatly improved the clarity and exposition of the paper.

\section{References}

A. M. Abouammoh and A. F. Mashhour (1983), On characterization of discrete unimodality: a survey, in: Developments in Statistics and its Applications, KSU Libraries, 327-342.

A. M. Abouammoh, A. M. Ali and A. F. Mashhour (1994), On characterizations and variance bounds of discrete $\alpha$-unimodality, Statist. Papers 35, 151-161.

A. M. Abouammoh and A. F. Mashhour (1994), Variance upper bounds and convolutions of $\alpha$-unimodal distributions, Statist. Probab. Lett. 21, 281-289.

M. H. Alamatsaz (1985), A note on an article by Artikis, Acta Math. Hungar. 45, $159-162$.

S. W. Dharmadhikari and K. Jogdeo (1986), Some results on generalized unimodality and an application to Chebyshev's inequality, in: Reliability and Quality Control, Elsevier, 127-132.

S. W. Dharmadhikari and K. Joag-Dev (1989), Upper bounds for the variances of certain random variables, Comm. Statist. Theory Methods 18, 3235-3247.

M. I. J a cobson (1969), The maximum variance of restricted unimodal distributions, Ann. Math. Statist. 40, 1746-1752.

J. Muilwijk (1966), Note on a theorem of M. N. Murthy and V. K. Sethi, Sankhyā Ser. B 28, 183.

S. E. Navard, J. W. Seaman and D. M. Young (1993), A characterization of discrete unimodality with applications to variance upper bounds, Ann. Inst. Statist. Math. 45, 603-614.

R. A. Olshen and L. J. Savage (1970), A generalized unimodality, J. Appl. Probab. 7, $21-34$.

J. C. W. Rayner (1975), Variance bounds, Sankhyā Ser. B 37, 135-138.

J. W. Seaman, P. L. Odell and D. N. Young (1985), Maximum variance for unimodal distributions, Statist. Probab. Lett. 3, 255-260.

J. W. Seaman, D. M. Young and D. W. Turner (1987), On the variance of certain bounded random variables, Math. Sci. 12, 109-116.

F. W. Steutel (1988), Note on discrete $\alpha$-unimodality, Statist. Neerlandica 42, 137-140.

D. M. Young, J. W. Seaman, D. W. Turner and V. R. Marco (1988), Probability inequalities for continuous unimodal random variables with finite support, Comm. Statist. Theory Methods 17, 3505-3519.

M. I. Ageel

Department of Mathematics

King Khalid University

Abha, P.O. Box 157, Saudi Arabia

E-mail: maqeel@ksu.edu.sa 